\title{
Process Monitoring Dataset from the Additive Manufacturing Metrology Testbed (AMMT): “Three-Dimensional Scan Strategies”
}

\author{
Brandon Lane and Ho Yeung \\ National Institute of Standards and Technology, \\ Gaithersburg, MD 20899, USA
}

brandon.lane@nist.gov

ho.yeung@nist.gov

Data DOI: https://doi.org/10.18434/M32044

Key words: additive manufacturing; laser powder bed fusion; selective laser melting.

Accepted: November 15, 2019

Published: November 19, 2019

https://doi.org/10.6028/jres.124.033

\section{Summary}

This document provides details on the files available in the dataset "20180708-HY-3D Scan Strategies" pertaining to a three-dimensional (3D) additive manufacturing (AM) build performed on the Additive Manufacturing Metrology Testbed (AMMT) by Ho Yeung on July 8, 2018. The files include the input command files, in-situ process monitoring data, and metadata. This data is the first of future planned “AMMT Process Monitoring Datasets," as part of the Metrology for Real-Time Monitoring of Additive Manufacturing project at the National Institute of Standards and Technology (NIST).

\section{Data Specifications}

\begin{tabular}{ll}
\hline NIST Operating Unit(s) & Engineering Laboratory, Intelligent Systems Division \\
\hline Format & $\begin{array}{l}\text { There are several types of data formats in this dataset. Please refer to } \\
\text { Section } 4 \text { for a description of all included data files. }\end{array}$ \\
\hline Instruments & $\begin{array}{l}\text { The Additive Manufacturing Metrology Testbed (AMMT) was used } \\
\text { to perform the laser scans. A Mikrotron EOSens 3CL }{ }^{1} \text { camera was }^{-}\end{array}$ \\
\hline
\end{tabular}

${ }^{1}$ Certain commercial equipment, instruments, or materials are identified in this paper in order to specify the experimental procedure adequately. Such identification is not intended to imply recommendation or endorsement by the National Institute of Standards and Technology, nor is it intended to imply that the materials or equipment identified are necessarily the best available for the purpose. 


\begin{tabular}{ll}
\hline & $\begin{array}{l}\text { used for co-axial melt pool monitoring, and a Basler acA3800-10gm } \\
\text { camera used for layer monitoring. Details are provided in Section 3. }\end{array}$ \\
\hline Spatial or Temporal Elements & These measurements were performed on July 8, 2018 \\
\hline Data Dictionary & N/A \\
\hline Accessibility & $\begin{array}{l}\text { All datasets }{ }^{2} \text { submitted to Journal of Research of NIST are publicly } \\
\text { available. }\end{array}$ \\
\hline License & https://www.nist.gov/director/licensing \\
\hline
\end{tabular}

\section{Experiment Method}

The general purpose of this experiment was to study the effect of varying laser scan strategies on the final 3D part quality. Details on the experiment design, results, and analyses will be provided in future publications; however, description of the associated data files and enough contextual information to use them is provided in this document. Figure 1 shows the part numbering and locations on the build plane. Each part utilizes a unique scan strategy, which includes variation of the scan path/geometry, as well as laser power and scan velocity. Each part has the same nominal $10 \mathrm{~mm}$ x $10 \mathrm{~mm}$ x $5 \mathrm{~mm}$ geometry which is provided in the stereolithography (STL) file "cube.stl" in the "Build Command Data.zip" folder, described in Section 4.

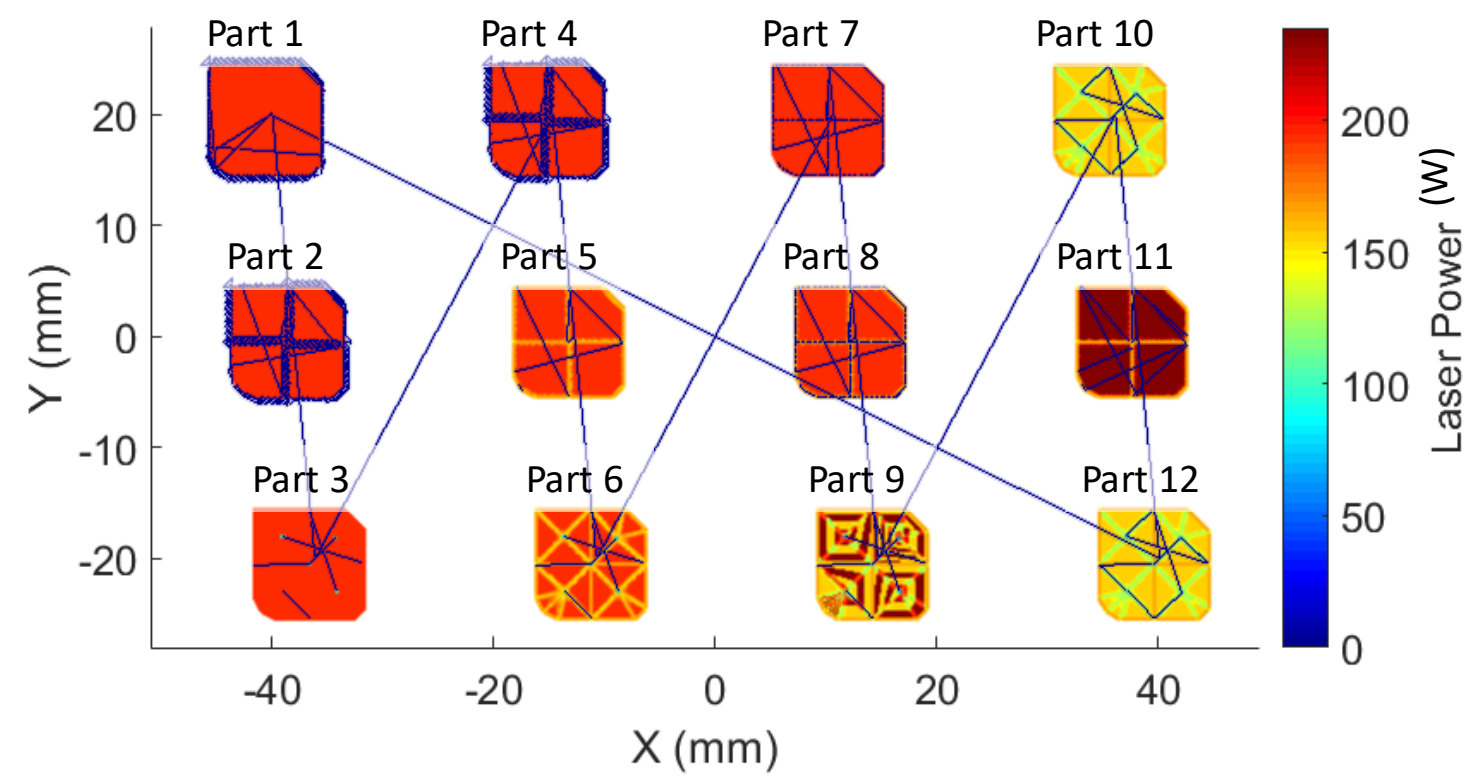

Fig. 1. Part numbering and layout for layer 1, showing relative difference in laser power.

In this experiment, melt pool monitoring (MPM) data was only captured for one part at each layer, such that Part 1 was captured while processing Layer 1, Part 2 captured during Layer 2, and so on. The cycle repeats every 12 layers, such that Part 1 is again captured during Layer 13. This was done by selectively triggering the MPM camera, described in Section 3.2.1, using the XYPT command files (which stands for X, Y, power, and trigger) described in Section 3.1.3. Examples of this pattern are readily

${ }^{2}$ The National Institute of Standards and Technology (NIST) uses its best efforts to deliver a high-quality copy of the Database and to verify that the data contained therein have been selected on the basis of sound scientific judgment. However, NIST makes no warranties to that effect, and NIST shall not be liable for any damage that may result from errors or omissions in the Database. 
observed in the example monitoring data synchronization videos discussed in Section 3.3. Layer image data, discussed in Section 3.2.2, was captured for every layer.

Coordinate systems are utilized throughout this document to describe location and orientation of the working components of AMMT and its instruments. The base coordinate system in the AMMT build plane follows ISO/ASTM 52921:2013 for a "generic (upward building) additive manufacturing system". The build volume origin is defined by the laser position when commanded to the point $(x, y)=(0,0)$, and the $\mathrm{z}=0$ position is defined by the recoater blade height when positioned above $(\mathrm{x}, \mathrm{y})=(0,0)$. Further discussion of this and other coordinate systems used to describe in-situ monitoring sensors are provided in Section 3.2.

\subsection{Process Inputs: Materials, Process Parameters, and Build Command}

\subsubsection{Materials and Scan Parameters}

The substrate material (that which is printed on) is a wrought nickel alloy 625 (IN625), hot rolled and annealed, and cut to $25 \mathrm{~mm} \times 100 \mathrm{~mm} \times 3.2 \mathrm{~mm}$ thick strips. The strips each had two $1 / 4-20$ tapped holes which mounts them to a base plate, and four were stacked next to each other. The materials certificate, which includes alloy composition, is provided with filename "20170619_In625_HPAlloys_0.25x1x4.pdf," located in the "Metadata.zip" folder.

The powder material is IN625. Powder compositions by weight measured by the vendor (using ASTM E1019 and ASTM E2823), as well as two third-party laboratories (using ASTM E1019) are provided in the tab-delimited text file "20180708_PowderComposition\&PSD.txt”. The powder particle size distribution is also included in this file, located in the "Metadata.zip" folder.

Since the objective of this study was to observe the effect of varying scan strategies, the scan parameters (laser power and speed) varied from part to part, and within each part. Intrinsic information regarding the scan parameters can be interpreted from the AM G-code or XYPT command files discussed in the following sections. However, solid layer height was consistently $20 \mu \mathrm{m}$, and laser spot diameter was $85 \mu \mathrm{m}$. Although process parameters changed continually throughout each part, the nominal scan speed was $800 \mathrm{~mm} / \mathrm{s}$, and the nominal laser power was $195 \mathrm{~W}$.

\subsubsection{AM G-Code}

The AM G-code files are stored in the "Build Command Data.zip” folder, and “AM Gcode” subfolder. Each part has an associated folder labeled "X_yyy_gcode," where $\mathrm{X}$ is the part number, and yyy is a description of the scan strategy. Within these folders, there is a *.gcode file (space-delimited text), a g-code generator parameter file, and a g-code interpreter parameter file, all of which are tab-delimited text files (except the *.gcode file) utilized by the testbed controller software.

The AMMT utilizes a custom-made laser powder bed fusion (LPBF) scan command development software called SAM [1,2]. The AM G-code files are being provided and stored for future reference by the authors and are not intended for use by outside users. However, these are used to generate the raw command files for the AMMT (the XYPT files), which contain all details of the 3D build scan strategy, including trigger timing for the melt pool monitoring camera.

\subsubsection{XYPT Files}

The XYPT command files are stored in the "Build Command Data.zip" folder, and "XYPT Commands" subfolder. The files are formatted as comma-separated value (.CSV) American Standard Code for Information Interchange (ASCII) text in four columns, where each file named "*layerXXXX.csv" provides the commands for layer number XXXX. 
The XYPT files provide the basic laser positioning and control commands for the AMMT. They are based on the XY2-100 command protocol for laser/galvo systems [1]. Figure 2 gives an example of the XYPT structure, and the resulting laser/galvo command. Each row represents a $10 \mu$ s timestep, resulting in a $100 \mathrm{kHz}$ digital command. The $\mathrm{X}(\mathrm{mm})$ and $\mathrm{Y}(\mathrm{mm})$ columns provide a position command for the laser/galvo. The power (W) column provides the laser power command.

The trigger column (unitless) is a decimal representation of the binary number which is used to fire the output trigger channels ( 8 channels labeled channels 0 through 7). For example, to trigger channel 1 (the second channel), binary representation is 0010 , decimal representation is ' 2 ', and so a ' 2 ' is in the 'Trigger' column in the XYPT file. To trigger both channel 0 and 2 (the first and third channels), binary representation is 0101 , decimal representation is 5 , and there would be a ' 5 ' in the trigger column. For this dataset, only channel 1 is connected to the melt pool monitoring camera; therefore a ' 2 ' in the T column indicates when a frame is captured.

\begin{tabular}{|c|c|c|c|}
\hline$X(\mathrm{~mm})$ & $Y(\mathrm{~mm})$ & Power $(\mathbf{W})$ & Trigger \\
\hline 0 & 0 & 0 & 0 \\
\hline 0.01 & 0 & 0 & 0 \\
\hline 0.02 & 0.01 & 100 & 2 \\
\hline 0.03 & 0.01 & 100 & 0 \\
\hline 0.04 & 0.02 & 0 & 2 \\
\hline
\end{tabular}
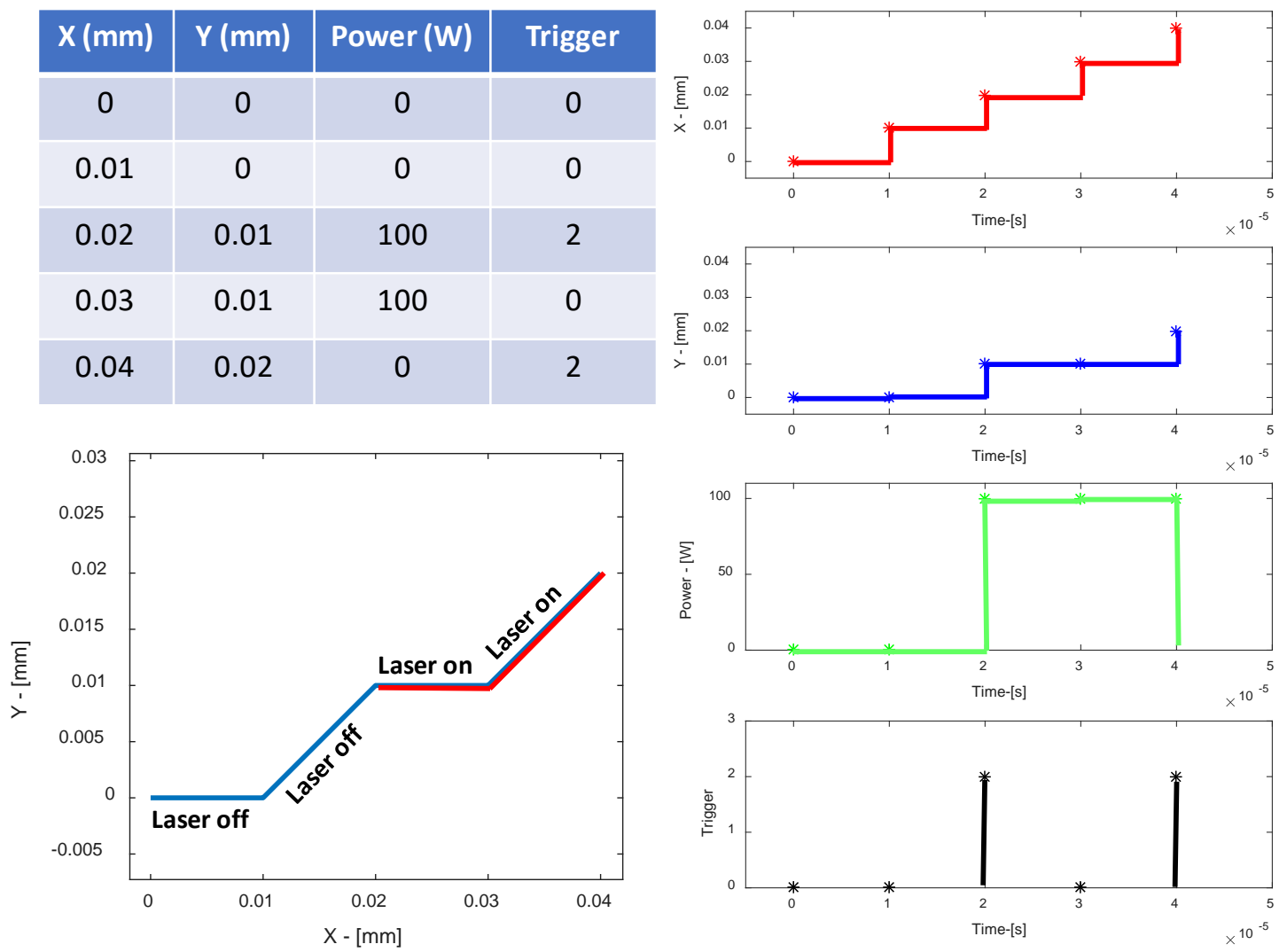

Fig. 2. Demonstration of the XYPT files. Upper left: Four-column data and units in the XYPT files. Lower left: Schematic showing the resulting commanded XY position of the laser and when the laser turns on. Right: Plots of each individual column's resulting effect on the AMMT. Note that the 'trigger' occurs for $20 \mu \mathrm{s}$, which is indicative of the camera integration time discussed in Section 3.2.1.

\subsection{In-situ Process Monitoring Data}

Data from two instruments are acquired during the 3D build: the co-axial MPM camera, and the layer camera. Figure 3 shows a general layout of the laser, galvos, MPM camera, and layer camera. The co-axial 
MPM camera is optically-aligned with the laser axis, such that the melt pool appears stationary in the field of view regardless of galvo XY position. The layer camera is at a fixed position above the build plane.

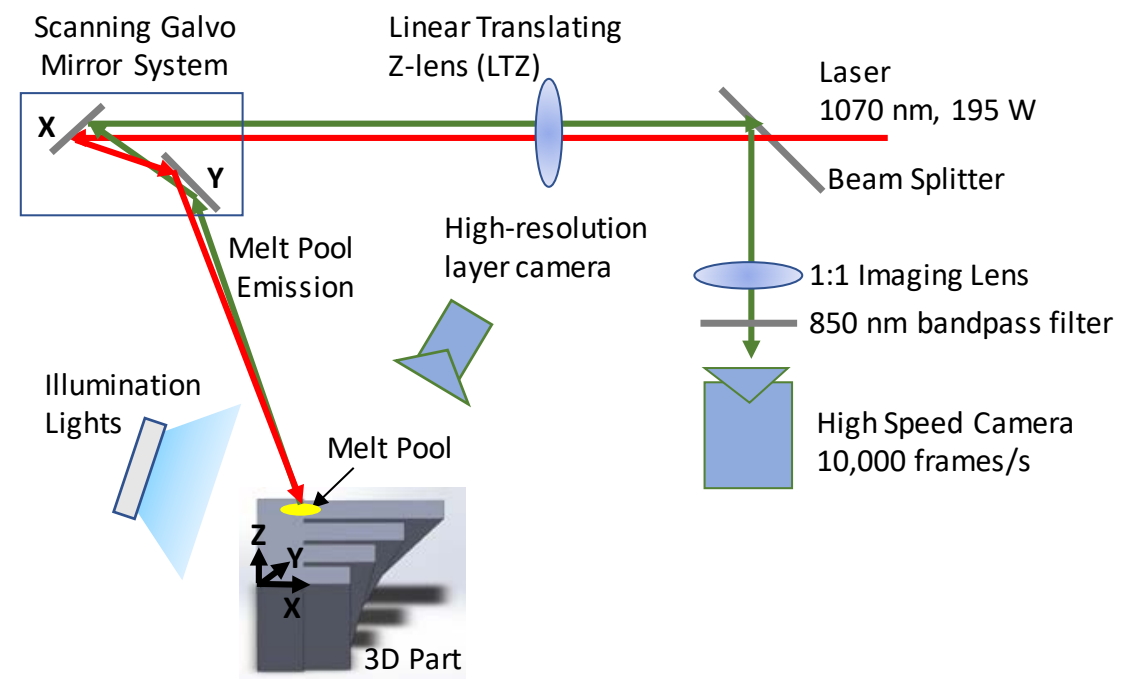

Fig. 3. Schematic of the AMMT 3D build process monitoring systems, including the high-resolution layer camera (layer imaging) and high speed MPM camera coaxially aligned with the laser.

To describe the orientation and positions of the co-axial MPM camera, or stationary instruments such as the layer camera, the following coordinates are defined and shown in Fig. 4. The laser spot position is defined by the vector ${ }^{\mathrm{A}} \mathrm{X}_{\text {laser, }}{ }^{\mathrm{A}} \mathrm{y}_{\text {laser }}>$ in the AMMT base coordinate system $\{\mathrm{A}\}$. A translating coordinate system $\{\mathrm{L}\}$ is tied to the laser spot with $\mathrm{x}, \mathrm{y}$, and $\mathrm{z}$ axes aligned with those of $\{\mathrm{A}\}$ (pure translation, no rotation).

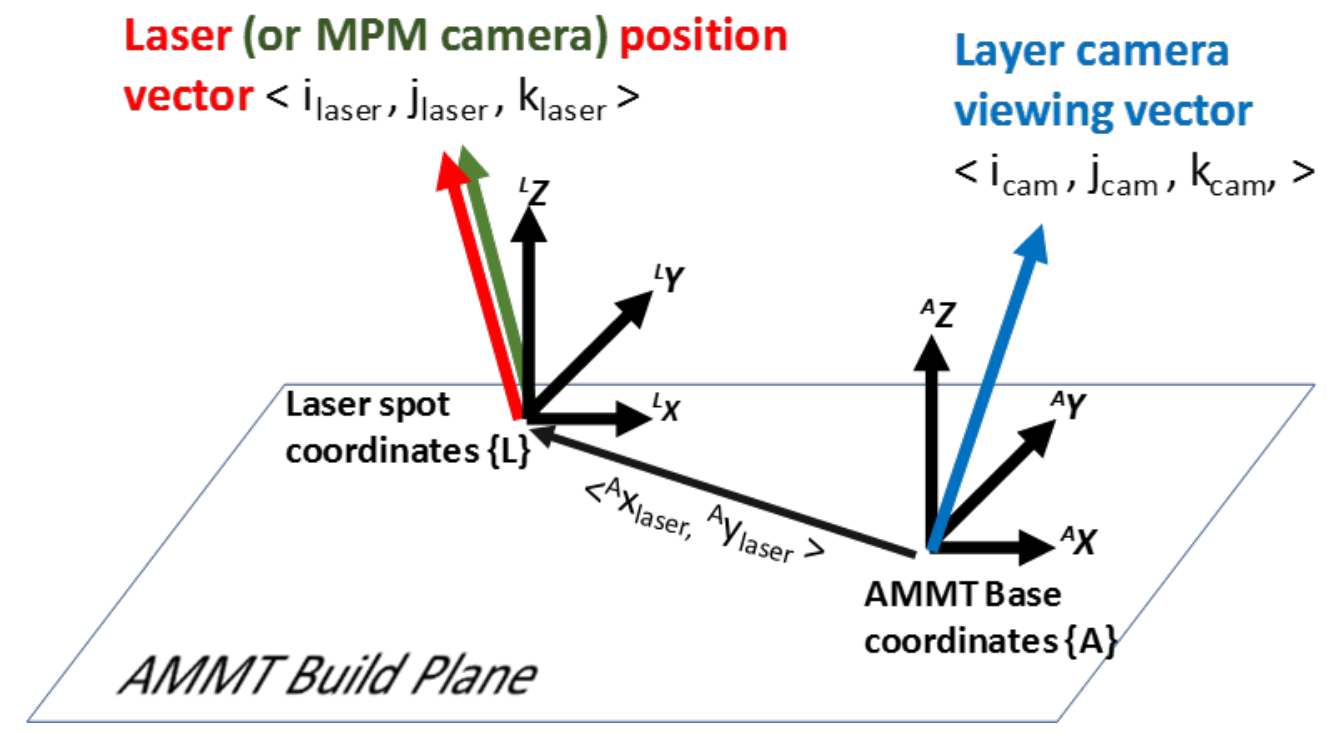

Fig. 4. Identification of coordinate systems and descriptive vectors. Orientation of stationary instruments (such as the layer camera) can be defined as a unit vector in the AMMT base coordinate system $\{\mathrm{A}\}$. The laser spot position in XY is defined by the vector $\left.<{ }^{\mathrm{A}} \mathrm{X}_{\text {laser, }}{ }^{\mathrm{A}} \mathrm{y}_{\text {laser }}\right\rangle$ in the AMMT base coordinate system $\{\mathrm{A}\}$. Laser angle/orientation can be defined by a unit vector in the laser spot coordinate system $\{\mathrm{L}\}$, which also defines the viewing angle of any co-axial instrument. 


\subsubsection{Co-axial Melt Pool Monitoring (MPM) Camera}

The co-axial melt pool monitoring (MPM) camera is optically aligned with the laser, such that the field of view of the camera is fixed to the laser spot coordinates $\{\mathrm{L}\}$ shown in Fig. 4, and the melt pool remains nominally stationary in the image. Additionally, the integration time (or shutter speed) was selected such that the median signal value (128 digital levels of an 8-bit digital range) equates approximately to the melt pool width measured in a-priori tests (approximately $100 \mu \mathrm{m}$ in Fig. 5). Table 1 provides operating characteristics of the MPM camera. The timing for each MPM image frame corresponds to a trigger point in the XYPT command files discussed in Section 3.1.3. Note that for this dataset, the camera was triggered $1.21 \mathrm{~ms}$ ( 121 points at $100 \mathrm{kHz}$ ) earlier than indicated in the XYPT file. The first $\approx 2$ frames (it was imaged 2000 frames per second, or $2 \mathrm{kHz}$ ) were taken before the laser was turned on, and last $\approx 2$ frames are missing at the end before the laser was switched off.

Table 1. Co-axial melt pool monitoring (MPM) camera parameters

\begin{tabular}{|c|c|}
\hline Camera Model & Mikrotron EOSens 3CL $^{1}$ \\
\hline Framegrabber Card & NI PCIe-1433 Cameralink Full ${ }^{1}$ \\
\hline Pixel Pitch of Detector & $8 \mu \mathrm{m}$ \\
\hline Window Size (H x V) & 120 pixels X 120 pixels \\
\hline Instantaneous Field of View (iFoV) & $8 \mu \mathrm{m} /$ pixel \\
\hline Magnification & $1 \mathrm{x}$ \\
\hline Viewing Angle & $\begin{array}{c}\text { Same as laser position vector } \\
\text { See Section 3.3: Metadata }\end{array}$ \\
\hline Frame Rate & Up to 10 000 Hz \\
\hline Integration Time / Shutter Speed & $20 \mu \mathrm{s}$ \\
\hline Bit depth & 8-bit (256 digital levels) \\
\hline Optical Filter Bandwidth & $850 \mathrm{~nm} \pm 20 \mathrm{~nm}$ \\
\hline
\end{tabular}

The object plane of the MPM imaging system is focused and occurs on the AMMT XY build plane; however, the image orientation does not align with the XY axes. Figure 5 demonstrates the relative orientation of the MPM images with three example image frames representing three laser scan directions. The left image is an example of an MPM image frame as it is acquired, with relative orientation to the AMMT shown. Note that image coordinates typically originate at the upper left corner, with the z-direction into the screen/page for a right handed coordinate system. The center image shows the relative orientation with z-direction out of the screen/page. The right-most image demonstrates the various vectors and translations. Equations (1) and (2) provide transformations between a pixel location ${ }^{\mathrm{M}} \vec{p}$ in MPM image coordinates $\{\mathrm{M}\}$ onto the AMMT base coordinates $\{\mathrm{A}\}$. Equation (1) translates the point ${ }^{\mathrm{M}} \vec{p}$ defined in image coordinate origin $\{M\}$, to an intermediate coordinate system $\{Q\}$, which is parallel to $\{M\}$, but has its origin at the center of the laser spot in the image.

$$
{ }^{\mathrm{Q}} \vec{p}=\left({ }^{\mathrm{M}} \vec{p}^{-\mathrm{M}} \vec{r}_{\text {laser }}\right) \cdot \mathrm{iFoV}
$$

The iFoV is the instantaneous field of view $(0.008 \mathrm{~mm} / \mathrm{pixel})$ for the MPM camera, which converts the pixel location point to units [mm]. The vector ${ }^{\mathrm{M}} \vec{r}_{\text {laser }}$ is the position of the laser center in [pixels] in the MPM image coordinate system $\{\mathrm{M}\}$, shown in the left-most frame in Fig. 5. Equation (2) provides the conversion between the pixel point ${ }^{\mathrm{Q}} \vec{p}$, to the AMMT base coordinates $\{\mathrm{A}\}$.

$$
{ }^{\mathrm{A}} \vec{p}={ }^{\mathrm{A}} \mathbf{R}_{\mathrm{Q}} \cdot{ }^{\mathrm{Q}} \vec{p}+{ }^{\mathrm{A}} \vec{r}_{\text {laser }}
$$

The matrix ${ }^{\mathrm{A}} \mathbf{R}_{\mathrm{Q}}$, shown in Fig. 5, rotates any point in $\{\mathrm{Q}\}$ to orient with $\{\mathrm{A}\}$. This rotation matrix may also be used to orient the melt pool orientation, measured in the MPM images, to the AMMT 
coordinates. The angle $\theta$ is the angle between the AMMT base coordinate system and the MPM image coordinate system, and the vector ${ }^{\mathrm{A}} \vec{r}_{\text {laser }}$ is the laser spot position, defined in $\{\mathrm{A}\}$, which is represented in the XYPT command files discussed in Section 3.1.3.
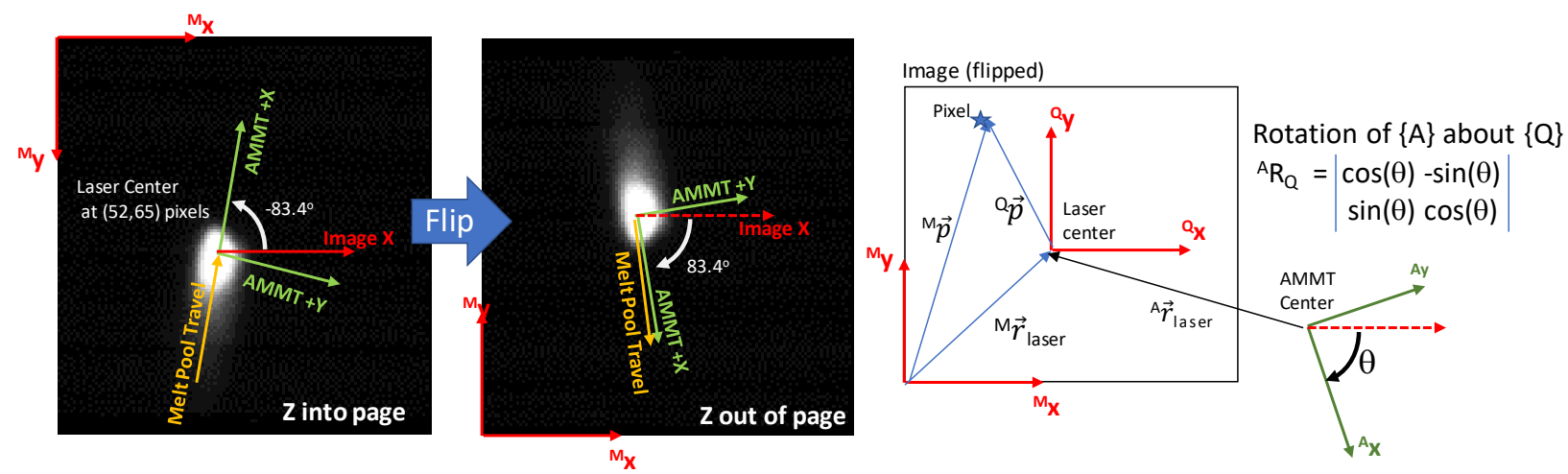

Fig. 5. Example co-axial MPM frames indicating the relative orientation with the AMMT. Left: Original frame indicating image coordinates $\{\mathrm{M}\}$, melt pool travel direction, and melt pool center. Center: flipped frame, which demonstrates how the image $\mathrm{Z}$ and AMMT z-directions align. Right: Vector and coordinate system definitions.

MPM camera orientation for this dataset was determined by examining over 20 image frames in which the laser was scanning in the $+X$ direction (AMMT base coordinates). Melt pool images were binarized at a threshold of 20 digital levels, and the orientation of second central moment of the binarized image calculated and averaged, resulting in $\theta=83.4^{\circ}\left(\sigma=0.8^{\circ}\right)$. The position of the laser in the MPM image ${ }^{\mathrm{M}} \vec{r}_{\text {laser }}$ was determined by selecting 20 frames in which the melt pool appeared very dim, such as when the laser is either turning on or off. This is hypothesized to be indicative of a point when the centroid of the dim spot aligns with the laser spot center. Similar to calculating the orientation, the images were binarized and centroid was calculated and averaged, which resulted in ${ }^{\mathrm{M}} \vec{r}_{\text {laser }}=(52,65)$ pixels.

The datafiles for the MPM camera are located in the "In-situ Meas Data.zip" folder, and the "Melt Pool Camera” subfolder. Subsequent subfolders, labeled "MIA_Lzzzz” where MIA represents "Melt pool Image Analysis” and zzzz is the layer number, contain the MPM image frames stored as bitmap (*.BMP) files, labeled “frameQQQQ.bmp,” where QQQQ is the frame number.

\subsubsection{Layer Imaging Camera}

The layer imaging camera, which is in a staring (fixed position) configuration, captured images of the build surface before and after recoating powder for each layer, as depicted in the experiment setup in Fig. 3. Two linear, light-emitting diode (LED) bars provided illumination in brightfield orientation. Table 2 provides the camera parameters for the layer imaging camera, as well as some information on the illumination sources. Figure 6 provides an example image, taken immediately after the laser scanned on layer 1 (the first powder layer). The camera position viewed through a plexiglass enclosure, which had a mounting hole that partially obstructed Parts 1 and 2. 
Table 2. Layer imaging camera parameters

\begin{tabular}{|c|c|}
\hline Camera Model & Basler acA3800-10. $\mathrm{gm}^{1}$ \\
\hline Pixel Pitch of Detector & $1.67 \mu \mathrm{m}$ \\
\hline Window Size $(\mathrm{H} \times \mathrm{V})$ & 2000 pixels $\times 2000$ pixels \\
\hline Instantaneous Field of View (iFOV) & $\begin{array}{l}\text { X: (63 to } 71) \mu \mathrm{m} / \text { pixel } \\
\text { Y: }(58 \text { to } 63) \mu \mathrm{m} / \text { pixel } \\
\text { See Fig. } 8\end{array}$ \\
\hline Magnification & Approx. 0.03x \\
\hline Viewing Angle (defined in $\{\mathrm{A}\}$ ) & $\mathrm{A}^{\mathrm{A}}<0.45,0,0.89>$ \\
\hline Frame Rate & $\begin{array}{l}2 \text { per layer (before powder spreading and } \\
\text { after) }\end{array}$ \\
\hline Bit depth & 8-bit (256 digital levels) \\
\hline Illumination Type & $\begin{array}{l}\text { Two Linear LED Bars, } 850 \mathrm{~nm} \text { wavelength } \\
\text { (Prophotonix LN1-850) }\end{array}$ \\
\hline Optical Filter Bandwidth & $850 \mathrm{~nm} \pm 20 \mathrm{~nm}$ \\
\hline
\end{tabular}

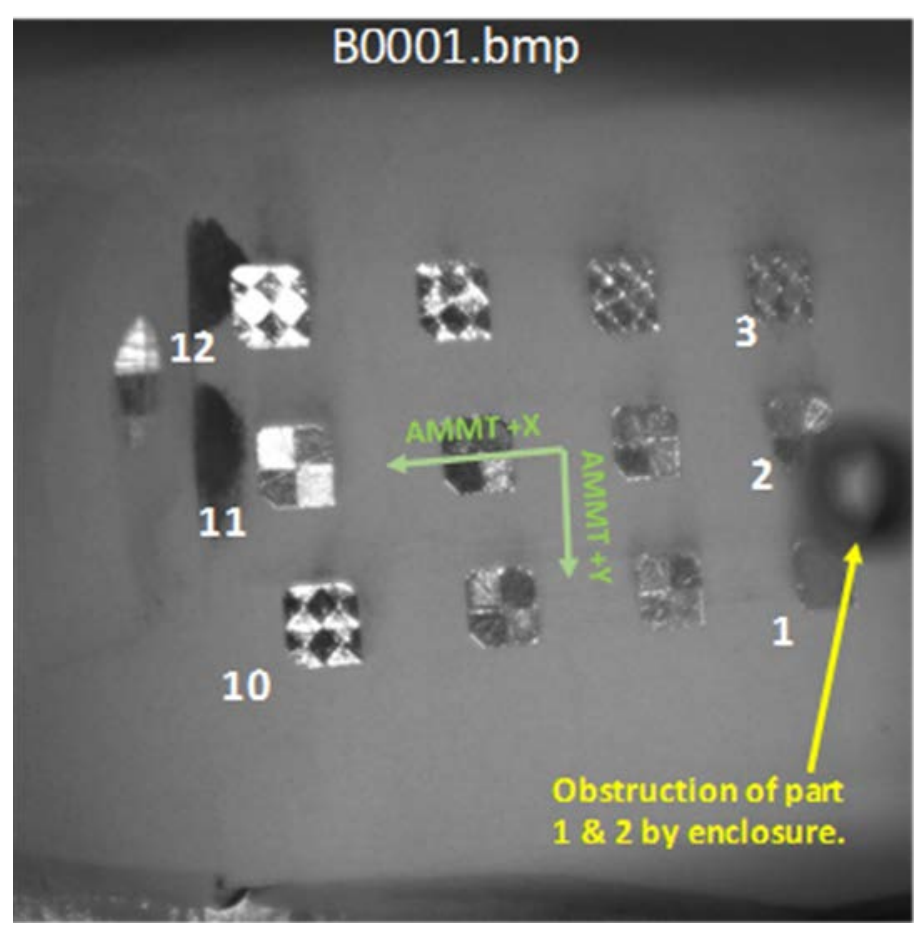

Fig. 6. Example layer image after the laser has scanned powder layer 1 (B0001.bmp). White numbers indicate the part number, and AMMT base coordinate system is shown.

The layer image file naming convention is based on whether they are captured after a layer of powder is spread (Axxxx) or after the laser has melted that layer (Bxxxx), where $\mathrm{xxxx}$ is the imaged layer. A simple way to remember: B0001 = 'Burned layer 1' or A0002 = 'After spreading layer 2'. Figure 7 gives an example sequence of images as they're captured in time, and their respective filenames. An example 'streak' caused by the recoating mechanism is created in image A0019 (after spreading layer 19), and is still visible in B0019 (burned layer 19). 

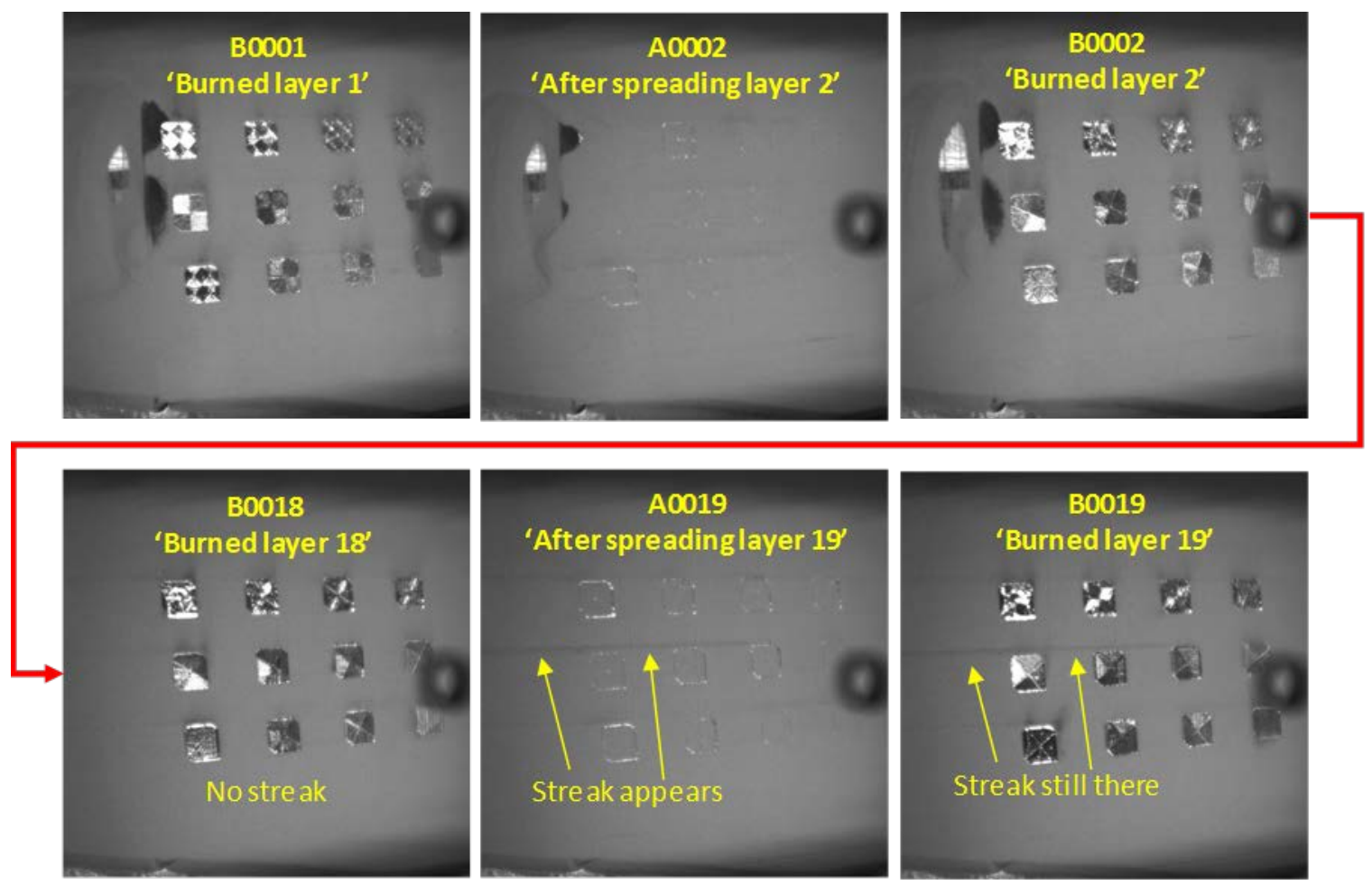

Fig. 7. Example sequence of layerwise images elucidating A (after spreading) and B (burned) naming convention.

A spatial calibration dot grid target was imaged prior to the 3D build to provide spatial calibration, as well as image distortion correction for better registration with the base coordinate system. The layer camera focus is manually adjusted such that the $(0,0,0)$ AMMT origin is in best focus. Illumination and camera settings for this calibration target were not the same as those used in the experiment, therefore brightness or intensity values should be ignored. The dots are $0.500 \mathrm{~mm}$ diameter, spaced $1.000 \mathrm{~mm}$ apart in 51 rows and 51 columns resulting in a $50 \mathrm{~mm} \times 50 \mathrm{~mm}$ grid. It is not known if the center dot (row 26, column 26) was aligned with the AMMT coordinate center, nor if the dot pattern was accurately oriented with the AMMT base coordinate system axes $\{\mathrm{A}\}$. 


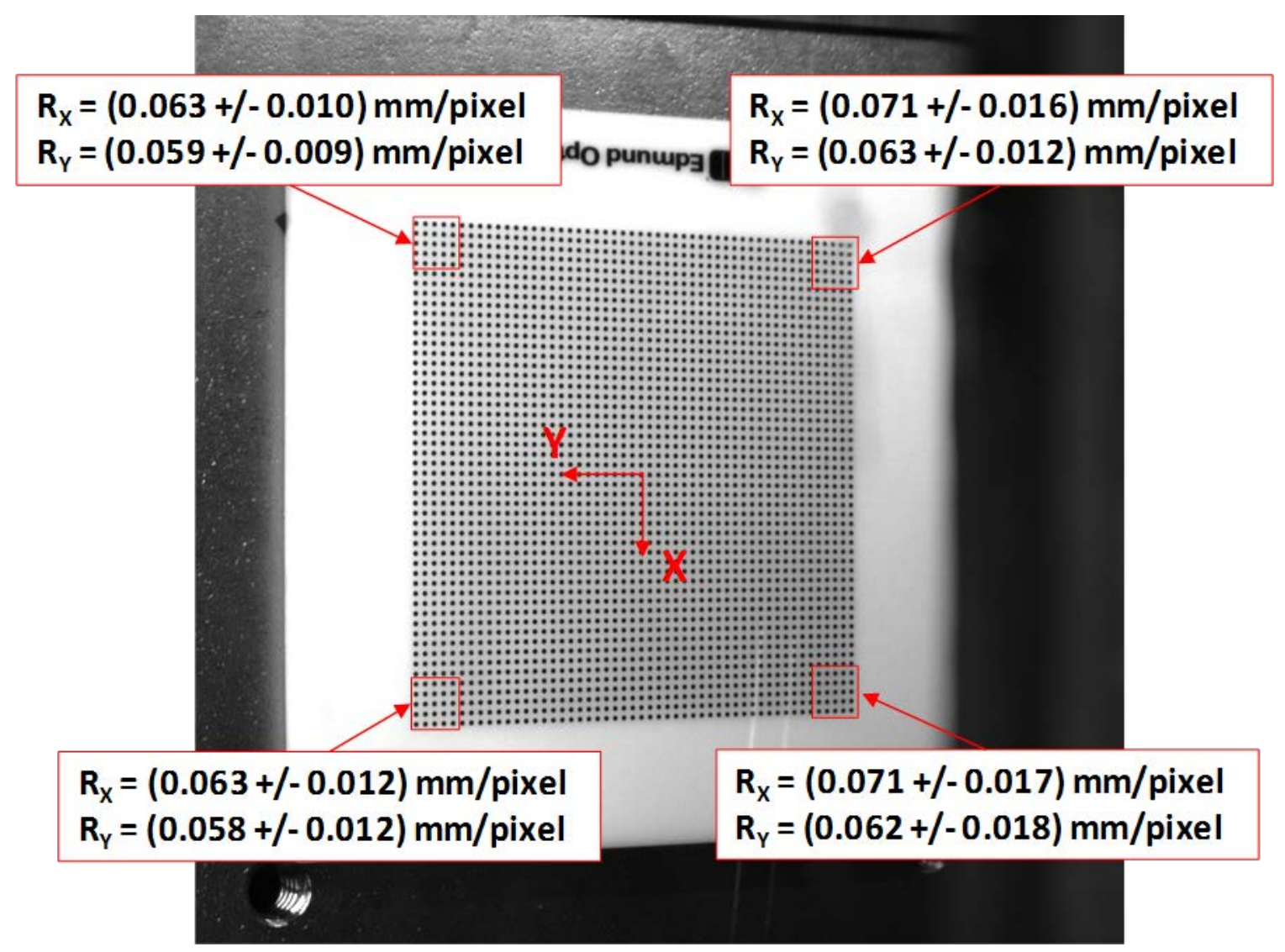

Fig. 8. Spatial calibration grid target observed with the layer imaging camera. It is not known if the dot grid center or orientation was aligned with the AMMT base coordinate system. Instantaneous field of view (iFoV) in the X and Y direction (Rx, Ry) was calculated from a $5 \times 5$ sub grid in each corner. iFoV values are given with \pm 1 standard deviation $(n=25)$

\subsection{Movies}

A select number of videos (in MP4 file format) were created that demonstrate the time-synchronization of the XYPT data with the co-axial MPM images, as well as provide qualitative description. Note that the 1.21 ms delay, detailed in Sections 3.1.3 and 3.2.1, was compensated for in these videos. These are stored in the "Movies.zip" folder and indicate in their respective file names which layer they correspond to. Figure 9 gives an example screenshot of one of these movies. In the upper-left, the MPM image frames are shown with melt pool width $[\mu \mathrm{m}]$, length $[\mu \mathrm{m}]$, and major axis orientation [degrees], in the upper left of the screenshot, and frame number in the lower right. Note that these images were rotated $100^{\circ}$ while processing to better, but not precisely, align with the machine base coordinates $\{\mathrm{A}\}$; therefore they do not align with the raw MPM image files. On the upper right, a scrolling timeseries chart shows melt pool area, calculated from the MPM images, along with the command laser power. The bottom plot shows the position of the laser within the AMMT build plane. 


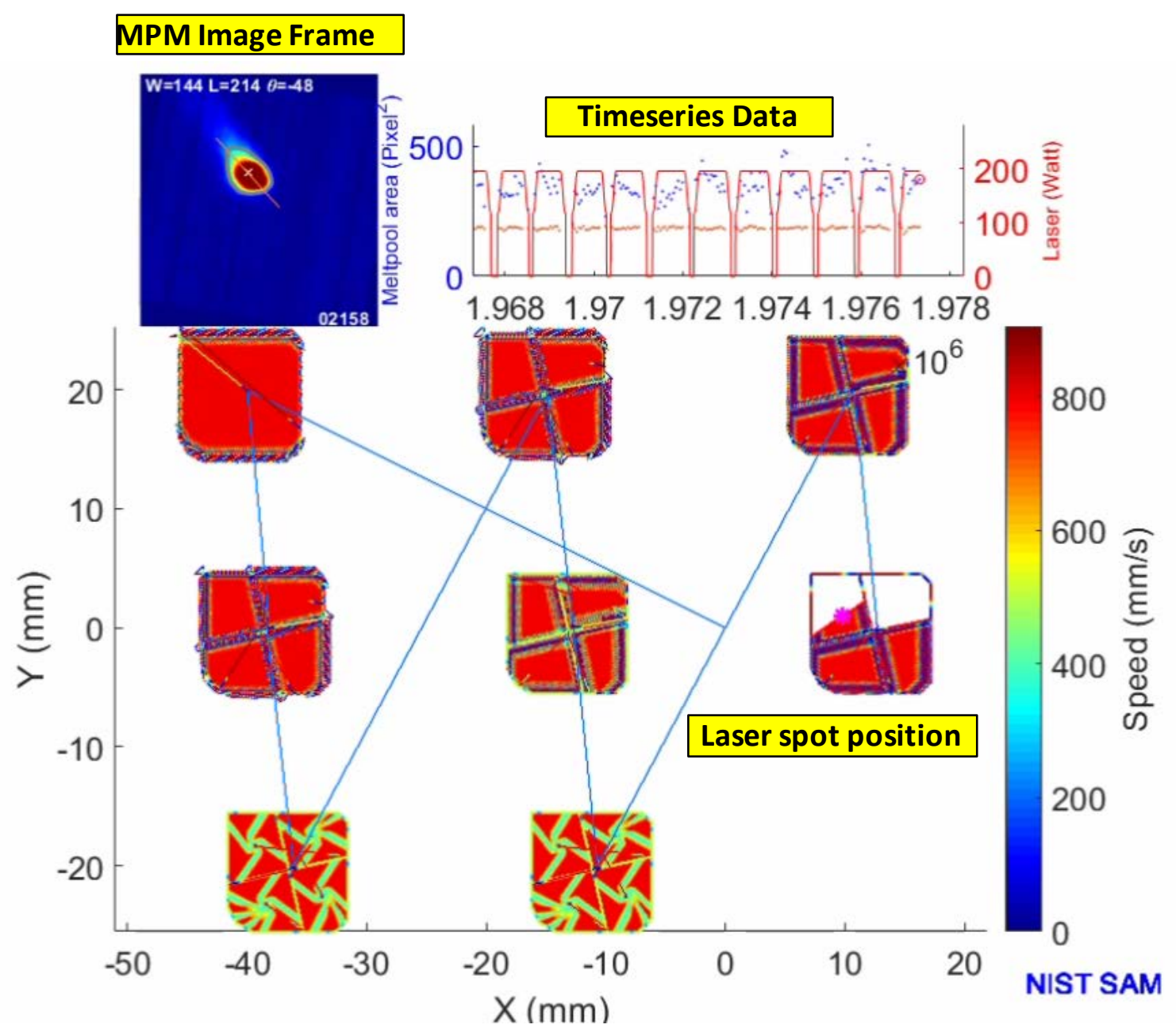

Fig. 9. Example screenshot from time-synchronized process monitoring data (layer 200).

\subsection{Metadata}

Several additional files pertaining to the system setup and materials are provided in the "Metadata.zip" folder. "2018_AMMTLaserScanAngles.txt" is a tab-delimited text file that provides the laser incident angle unit vectors with respect to the build plane as shown in Fig. 4. Three tables, representing $\mathrm{i}_{\text {laser, }}$ jlaser, and $\mathrm{k}_{\text {laser }}$ vector components, are provided vs. laser positions in the AMMT base coordinate system, where the top row and left column are the positions of ${ }^{\mathrm{A}} \vec{r}_{\text {laser }}$ in [mm]. Figure 10 gives an example quiver plot showing the results, where $\mathrm{i}_{\text {laser }}$ and $\mathrm{j}_{\text {laser }}$ magnitudes are amplified $5 \mathrm{x}$ to demonstrate how the laser angle varies.

Also included in the "Metadata.zip" folder are the layer camera dot grid calibration image shown in Fig. 8, the material certificates for the IN625 powder and substrate materials, and a tab-delimited text file providing the IN625 powder composition measured by the vendor and two outside laboratories, as well as the D10, D50, and D90 values of particle size distribution. 


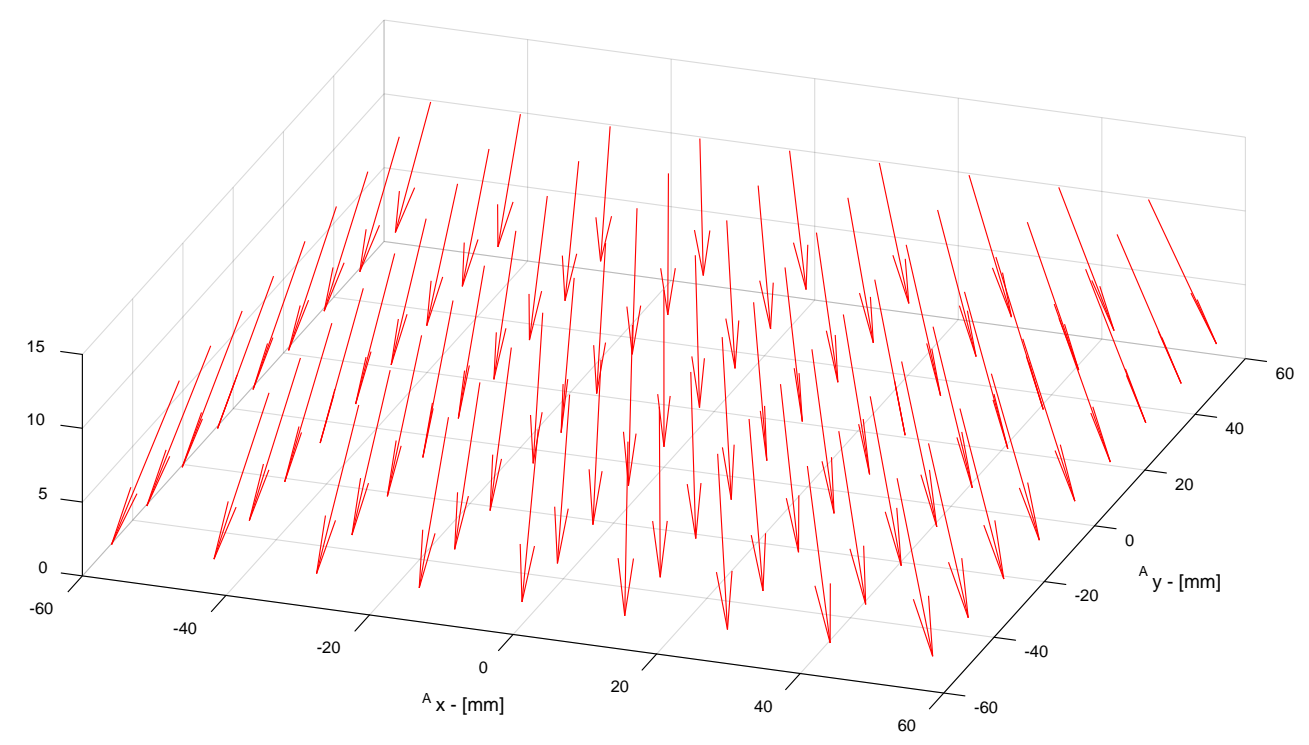

Fig. 10. Laser orientation $<\mathrm{i}_{\text {laser }}, \mathrm{j}_{\text {laser }}, \mathrm{k}_{\text {laser }}>$, mapped to the corresponding ${ }^{\mathrm{A}} \vec{r}_{\text {laser }}$ position in the AMMT base coordinates. The $\mathrm{i}_{\text {laser }}$ and $\mathrm{j}_{\text {laser }}$ magnitudes are amplified $5 \mathrm{x}$ in this plot to highlight the laser angle.

\section{Data Files}

The dataset contains the following files:

- Lane-JResData-3DScanStrategies.pdf

o This dataset overview document.

- Build Command Data.zip

o Subfolder: AM Gcode

- Note that the AM Gcode is not intended for general use, but is maintained in this dataset for archival purposes.

- Individual subfolders are labeled "X_cube_YYYY," where $\mathrm{X}$ is the part number as shown in Fig. 1, and YYYY is a description of the scan strategy.

- $\quad$ File: X_cube_YYY.gcode: ASCII text file with AM Gcode

- File: Gcode_interpreter_par.csv: ASCII text file with AM Gcode interpreter parameters.

- File: Gcode_generator_par.csv: ASCII text file with AM Gcode generator parameters.

o Subfolder: XYPT Commands

- Files: ZZZZZZZ_layerXXXX.csv: Comma-separated text file, where ZZZZ is file name resulting from the G code software, and XXXX indicates the layer.

o cube.STL

- A Stereolithography (STL) file representing the 3D geometric data of the individual parts.

- In-situ Meas Data.zip

o Subfolder: Melt Pool Camera 
- Subfolders: MIA_LXXXX: Each subfolder represents co-axial melt pool camera images for layer XXXX, where XXXX ranges from layer 0001 to 0250.

- Files: Each subfolder contains a list of bitmap (BMP) figures with the file notation "frameYYYYY.bmp," where YYYYY represents a video frame number.

- Note that the timing of each video frame corresponds to a trigger indicated in the corresponding layer's XYPT file, albeit with a $1.21 \mathrm{~ms}$ (121 points) delay (i.e. the MPM camera actually triggered $1.21 \mathrm{~ms}$ before indicated in the XYPT file.

o Subfolder: Layer Camera

- Files: 8-bit grayscale bitmap images, labeled 'AXXXX.bmp' or 'BXXXX.bmp' representing new powder layer (A) or recently melted (B) layers, where XXXX indicates the layer. Refer to Section 3.2.2.

- Metadata.zip

o File: 2018_AMMTLaserScanAngles.txt

- A tab-delimited text file providing the components of a unit vector representing the laser incident angle as represented in Fig. 10. $\mathrm{X}$ and $\mathrm{Y}$ laser position values are in $[\mathrm{mm}]$.

o File: 201807_LayerCamera_DotGrid.bmp.

- 8-bit grayscale bitmap image of a dot grid target as shown in Fig. 8.

o File: 20170619_In625_HPAlloys_0.25x1x4.pdf

- Material certificate for the IN625 substrate material.

o AMB2018-01_625_MaterialCertificate.pdf

- Material certificate for IN625 metal powder.

o 20180708_PowderComposition\&PSD.txt

- Tab-delimited text file of IN625 metal powder element composition, indicated in columns.

- $\quad$ Movies.zip

o Files: YYYYY_layerXXXX.mp4

- MP4 video files of the example synchronized process monitoring data shown in Fig. 9, where YYYYY is a file prefix and XXXX indicates layer number.

\section{Impact}

This dataset provides open access, industrially-relevant AM in-situ process monitoring data for researchers to apply various image or data processing or machine learning algorithms. This process monitoring data is otherwise inaccessible on commercial AM machines, or lacks description of key calibration or characterization metadata as provided here. This dataset is part of the NIST Metrology for Real-Time Monitoring of Additive Manufacturing project.

\section{References}

[1] Yeung H, Neira J, Lane B, Fox J, Lopez F (2016) Laser path planning and power control strategies for powder bed fusion systems. Proceedings of the 27th Annual International Solid Freeform Fabrication Symposium, Austin, TX. 113-127. https://sffsymposium.engr.utexas.edu/sites/default/files/2016/008-Yeung.pdf

[2] Yeung H, Lane BM, Donmez MA, Fox JC, Neira J (2018) Implementation of advanced laser control strategies for powder bed fusion systems. Procedia Manufacturing (26):871-879. https://doi.org/10.1016/j.promfg.2018.07.112. 
About the authors: Brandon Lane leads the Metrology for Real-Time Monitoring of Additive Manufacturing Processes project, part of the Measurement Science for Additive Manufacturing program in the NIST Engineering Laboratory. Ho Yeung leads the Machine and Process Control Methods for Additive Manufacturing project, part of the Measurement Science for Additive Manufacturing program in the NIST Engineering Laboratory. The National Institute of Standards and Technology is an agency of the U.S. Department of Commerce. 